\title{
Assessment of direct medical cost using cost of illness analysis in patients with dengue fever - Retrospective study
}

\author{
Shaikh Rafiya Rafikahmed ${ }^{\mathrm{a}}$, Uday Venkat Mateti ${ }^{\mathrm{a}, *}$, Chethan Subramanya ${ }^{\mathrm{b}}$, Shraddha Shetty ${ }^{\mathrm{c}}$, \\ Anu Sunny ${ }^{\mathrm{a}}$, Anjana Madhusoodanan ${ }^{\mathrm{a}}$ \\ ${ }^{a}$ Nitte (Deemed to be University), NGSM Institute of Pharmaceutical Sciences, Department of Pharmacy Practice, Deralakatte, Mangaluru, Karnataka, India \\ ${ }^{\mathrm{b}}$ Nitte (Deemed to be University), KS Hegde Medical Academy, Justice KS Hegde Charitable Hospital, Department of General Medicine, Deralakatte, Mangaluru, \\ Karnataka, India \\ ${ }^{\mathrm{c}}$ Nitte (Deemed to be University), KS Hegde Medical Academy, Department of Biostatistics, Deralakatte, Mangaluru, Karnataka, India
}

\section{A R T I C L E I N F O}

\section{Keywords:}

Dengue

Fever

Cost of illness

Disease burden

Medical cost

\begin{abstract}
A B S T R A C T
Introduction: Dengue fever is a mosquito-borne human viral disease spread globally and is regarded as a global threat by the World Health Organization (WHO). In India, an appalling rise has recently been observed, resulting in an economic burden.

Objective: To study the direct medical costs using cost of illness analysis and the varied clinical presentations of the disease.

Methodology: A retrospective study was conducted among patients diagnosed with dengue fever in the general medicine department. Data was collected from eligible dengue patients admitted between June 2018 to December 2019. Patient's demographic details, clinical presentations, factors affecting the cost, direct medical cost details were recorded.

Results: The study enrolled a total of 220 patients., with $75 \%$ being males and $25 \%$ being females. The majority of the patients (44.54\%) were between the ages of 19-30. The average length of hospital stay was found to be 4.55 days, with the highest number of cases occurring in June and July. The NS1 antigen test yielded 88.63\% positive results, and males had a higher total median direct cost of USD 102.83 than females, USD 99.16. The age group 46-60 years had the highest total median direct cost of USD 135.16 compared to other age groups.

Conclusion: According to the findings, the median total direct medical cost was USD 119.29. The laboratory charges incurred $34.02 \%$ of the total cost, consultation charges incurred $17.18 \%$ of the total cost, and medication charges incurred $14.72 \%$ of the total cost.
\end{abstract}

\section{Introduction}

Dengue fever, also known as break-bone fever, is one of the most common mosquito-borne viral infections that has rapidly spread throughout the world and is regarded as a global threat by the World Health Organization (WHO). ${ }^{1}$ Unplanned urbanization, environmental changes, climatic factors, population immunity levels, socio-economic and man-made environmental changes were the factors in the disease spread. Although dengue incidence is generally underreported in developing countries due to inadequate surveillance and monitoring systems, the current estimates of the WHO show that the number of cases reported has increased by more than eightfold over the last two decades from 2.4 million in 20104.2 million in 2019. Every year, India is afflicted with a dengue outbreak. According to the National Vector Borne Disease Control Programme (NVBDCP) 2018 report, the reported dengue cases have dramatically increased from 10,137 during the 2006-2008 period to $1,88,401$ cases in $20171,57,315$ cases were recorded in 2019. ${ }^{2,3}$

Dengue fever is an infectious disease spread by the bite of an infected female Aedes Aegypti or Aedes Albopictus mosquito from the Flaviviridae family. The clinical characteristics of dengue fever have rapidly changed in recent years, with the clinical profile ranging from selflimiting febrile illness to severe dengue and life-threatening conditions. ${ }^{4,5}$ India experiences an outbreak of dengue fever, resulting in a significant financial burden on both the government and the patients. Although no specific treatment has been discovered yet, prevention and

\footnotetext{
* Corresponding author.

E-mail address: udayvenkatmateti@gmail.com (U.V. Mateti).
} 
control efforts have achieved success to a limited extent. ${ }^{5}$

When resources are limited, Pharmacoeconomic evaluations can be an important tool in making therapeutic decisions. The cost of illness (COI) is also known as Burden of Illness (BOI). It encompasses several aspects of illness as well as their impact on health outcomes in a country, a specific region, societies, and individuals. COI study examines the direct and indirect costs of a disease or its risk factors. The goal of COI is to determine the level of the economic burden imposed by patients; as a result, the economic costs and burden of disease must be quantified. To avoid complications, atypical clinical manifestations of the disease should also be considered. Because only a few studies are available in India, the current need for the study is to learn about different clinical presentations and estimate the economic burden. Economic studies are critical for understanding the BOI and assisting in the planning of health services and policy. The Pharmacoeconomic study, in which the costs and consequences of pharmaceutical products and services are measured and compared, can reduce the economic burden and improve the quality of life. Therefore, this study was designed to investigate clinical presentations due to different clinical features and evaluate the direct medical cost in dengue fever patients, providing data that may be useful in effectively managing the disease. ${ }^{6-8}$

\section{Methodology}

\subsection{Study design, setting and duration of the study}

A retrospective study design was conducted in the general medicine unit of a tertiary care teaching hospital. The study analyzed the medical records of eligible dengue patients from June 2018 to December 2019.

\subsection{Sample size}

The minimum sample size was determined to be 220. Sample size was arrived by using the formula: Expected proportion of patients with dengue fever $=0.024$; expected precision $=2 \%$; confidence interval at $95 \%$.

$n=\frac{Z_{1-\alpha / 2}^{2} \boldsymbol{p q}}{d^{2}}$

$$
\begin{aligned}
& \mathrm{p}=0.024 \\
& \mathrm{q}=0.976 \\
& \mathrm{~d}=0.02 . \\
& \mathrm{Z}_{1-\alpha / 2}=1.96 \\
& \mathrm{n}=220
\end{aligned}
$$

\subsection{Ethical permission and registry}

Permission was obtained from the Institutional Ethics Committee (IEC) (Ref no: NGSMIPS/IEC/O2/2020) and Clinical Trials RegistryIndia CTRI/2020/12/029749 before starting the study.

\subsection{Study criteria}

Patients admitted to the general medicine department with age above 18 years and diagnosed with dengue fever and who had been hospitalized for at least two days were included in the study. Pregnant women, patients with incomplete medical records, and co-morbid conditions cases were excluded from the study.

\subsection{Data collection tools}

Patient information was gathered using patients' treatment charts, the hospital billing system, hospital pharmacy records, and patient medical records.

\subsection{Data collection procedure}

The samples were collected using the convenience sampling method, and the data was recorded from the day of admission until the day of discharge. Patient demographic parameters such as age, gender, social habits, laboratory data, length of hospital stay, health care schemes and signs and symptoms were recorded. The pharmacoeconomic evaluation took into account length of stay, laboratory charges, medication charges, consultation fees, nursing charges, and hospital charges. The data observed was analyzed for the average cost incurred in treating the dengue fever patients and was calculated based on patients' total direct medical cost. The Indian Rupees (INR) cost was converted to US Dollars (USD) at the rate of 1 USD $=72.80$ INR.

\subsection{Statistical analysis}

The data variables such as age group, gender, social habits, health care schemes, and clinical presentations are presented in frequency and percentage. The cost data is presented in median and IQR (Q3-Q1). Mann Whitney $U$ test analyzed the difference in total costs and gender, healthcare scheme, and length of hospital stay. The Kruskal-Wallis test analyzed the difference in total costs and age groups, and type of wards. The p-value $<0.05$ was conceded as statistically significant.

\section{Results}

\subsection{Demographic analysis}

A total of 220 confirmed cases were enrolled in the study, with 164 (75\%) males and 56 (25\%) being females. The age group of 19-30 years reported the highest number of cases. (44.54\%), followed by 78 cases (35.46\%) from 31 to 45 years. Most of the cases occurred in June-July (97 cases), and 195(88.63\%) cases were found positive for the Nonstructural protein one antigen test compared to antibodies test. Around $20 \%$ of cases were found to have social habits such as drinking alcohol or smoking or chewing substances. The details are summarized

Table 1

Demographic profile, Length of hospital stay and Dengue card test

\begin{tabular}{ll}
\hline Demographic characteristics & \\
\hline Distribution based on gender & \\
Gender & Frequency (\%) $(\mathbf{n}=\mathbf{2 2 0})$ \\
$\quad$ Male & $164(75)$ \\
Female & $56(25)$ \\
Distribution based on Age groups & \\
Age groups & Frequency $(\mathbf{n}=\mathbf{2 2 0})(\%)$ \\
$19-30$ & $98(44.54)$ \\
$31-45$ & $78(35.45)$ \\
46-60 & $44(20)$ \\
Distribution based on social habits & \\
Social Habits & Frequency $(\mathbf{n}=\mathbf{2 2 0})(\%)$ \\
No Habits & $179(81)$ \\
Alcohol & $19(9)$ \\
Smokers & $11(5)$ \\
Substance & $4(2)$ \\
Both Alcohol and Substance/Smoking & $7(3)$ \\
Distribution based on Length of hospital stay & \\
Number of days & Frequency (n= 220) (\%) \\
3 & $40(18.18)$ \\
4 & $79(35.90)$ \\
5 & $48(21.81)$ \\
6 & $45(20.45)$ \\
7 & $8(3.63)$ \\
Distribution based on Dengue Card Test & \\
Types & Frequency (n= 220) (\%) \\
Non-structural protein1 (NS1) antigen test & $195(88.63)$ \\
Immunoglobulin G (IgG) antibody test & $11(5)$ \\
Immunoglobulin M (IgM) antibody test & $11(5)$ \\
NS1 \& IgM & $2(0.90)$ \\
NS1 \& IgG & $1(0.45)$ \\
\hline &
\end{tabular}


in Table 1.

\subsection{Clinical presentation analysis}

Fever with and without chills was observed in $100 \%$ of cases and lasted for 3-5 days. As summarized in Table 2, other clinical features included vomiting (59\%), headache (40\%), loose stools (21.81\%), generalized weakness (20.45\%), Myalgia (20\%), etc. A rapid decrease in platelet count and WBC count was observed, which improved after treatment. Antipyretics and anti-biotic, anti-emetic, hepato-protective, anti-diarrheal, GI protectants, IV fluids, and nutritional supplements were administered for treatment. Platelet transfusion was performed in 50 cases $(22.52 \%)$ where the platelet counts were found to be less than 40000 cells $/ \mathrm{mm} 3$ on consecutive days. The average length of stay in the hospital was discovered to be $4.55 \pm 1.11$ days.

\subsection{Direct medical cost analysis}

The total median direct medical cost was found to be USD 119.89 (INR 8728.50) in the current study, as summarized in Table 3. The median direct medical cost for laboratory charges USD 40.80 (INR 2970) was the highest among the various cost components, followed by the other charges, as summarized in Table 3. Costs were also classified based on age groups, with the total median direct medical cost for the age group 46-60 years USD 135.16 (INR 9850) being the highest when compared to other age groups, as shown in Table 4. It was also seen that the total median direct medical cost for males was found to be USD 102.83 (INR 7495) and for females USD 99.16 (INR 7227.50). Accordingly, when the cost was compared with the wards, it was seen that the highest total median cost was charged for the rooms with AC USD 160.22 (INR 11676) when compared to the other rooms as summarized in Table 4. In this study, few patients (36\%) had schemes, with the median direct medical cost being highest for patients with MED USD 207.91 (INR 15152.5) and SVT scheme USD 207.91 (INR 15152.5). Table 4 describes the overall median direct medical cost and IQR (Q3Q1) values.

\section{Discussion}

As Dengue is emerging as a serious health problem in India, it is evident that there must be a rapid increase in the number of cases and changes in the characteristics such as epidemiology, demographic characteristics, clinical profile, and BOI of Dengue Fever. Because there are few studies available on the Clinical profile and economic burden of Dengue fever in India, this study was undertaken to learn more about it, which may be proven helpful for disease management.

During the study period, a total of 220 patients were considered. Male patients (75\%) were more than female patients in the current study

Table 2

Clinical presentations.

\begin{tabular}{ll}
\hline Clinical Features & Frequency $(\mathrm{n}=220)(\%)$ \\
\hline Fever & $94(42.72)$ \\
Fever with Chills & $126(52.27)$ \\
Nausea & $29(13.18)$ \\
Vomiting & $130(59)$ \\
Headache & $88(40)$ \\
Body Pain & $47(21.36)$ \\
Malaise & $16(7.27)$ \\
Loose Stools & $48(21.81)$ \\
Generalized Weakness & $45(20.45)$ \\
Myalgia & $44(20)$ \\
Arthralgia & $17(7.72)$ \\
Rashes & $30(13.63)$ \\
Cough & $26(11.81)$ \\
Cold & $22(10)$ \\
Giddiness & $7(3.18)$ \\
\hline
\end{tabular}

Table 3

Direct medical costs analysis.

\begin{tabular}{llllll}
\hline \multirow{2}{*}{ Cost category } & \multicolumn{2}{l}{ Cost (USD) } & & \multicolumn{2}{l}{ Cost (INR) } \\
\cline { 2 - 3 } \cline { 5 - 6 } \cline { 5 - 6 } & Median & IQR (Q3-Q1) & & Median & IQR(Q3-Q1) \\
\hline Medication cost & 17.56 & $33.05-10.97$ & & 1278.50 & $2405.75-798.75$ \\
Laboratory cost & 40.80 & $55.25-32.23$ & & 2970 & $4022.50-2346.25$ \\
Consultation cost & 20.60 & $27.47-14.56$ & & 1500 & $2000-1060$ \\
Nursing cost & 13.74 & $17.17-10.30$ & & 1000 & $1250-750$ \\
Hospital cost & 20.60 & $40.03-5.08$ & & 1500 & $2913.75-370$ \\
Miscellaneous cost & 6.59 & $9.72-0.09$ & & 480 & $707.50-330$ \\
Total cost & 119.89 & $139.85-70.74$ & & 8728.5 & $10180-5148.75$ \\
\hline
\end{tabular}

(25\%). A similar result was observed in the study conducted by Jain D et al., in Haryana, where $63.3 \%$ of the patients were males, and $36.7 \%$ were females. ${ }^{9}$ Demographic analysis reveals that most patients are between the ages of 19-30 (44.54\%). This is consistent with the findings of a study conducted by Dinkar A et al., where most patients were between the ages of $20-30(50.22 \%) .{ }^{10}$ The current study found that most cases occurred in the months of June-July $(n=97)$. In contrast, the study conducted by Singh $\mathrm{J}$ et al., in North India found that the most cases occurred in the month of August-September $(n=141) .{ }^{5}$ Based on Clinical Presentations, most of the patients were found to have a fever with chills (52.27\%) and fever (42.72\%). Apart from fever, the other symptoms were observed, such as headache (40\%), vomiting (59\%), myalgia (20\%), rashes (13.63\%), etc. A similar study was conducted by Kale AV et al., in a hospital in Maharashtra and showed similar findings. ${ }^{11}$ The mean length of hospital stay (LOHS) was $4.55 \pm 1.11$ days in the current study. According to a similar study conducted by VO NTT et al., in Vietnam, the mean LOHS was discovered to be $4.88 \pm 1.98$ days, which was similar to our findings. ${ }^{12}$

Based on Direct Medical cost, the median direct medical total costs for 220 patients were estimated to be USD 101.51 (IQR139.85-70.74). The result was in contrast with the similar study conducted by Panmei $\mathrm{K}$ et al., for 391 adults, and the median direct medical total costs were USD 287.22 (IQR 389.34-210.96). ${ }^{13}$ Based on the scheme, the mean direct medical cost for non-insurance and insurance was INR 8410.80 (USD 115.53 ) and INR 8170.68 (USD 112.20), respectively. The results were in contrast with the study conducted by Supdami W et al., in Indonesia, where the mean direct medical costs for insurance and non-insurance were US\$ 251.29 and US\$ 318.83, respectively. Compared to our findings, the economic burden was high in the study conducted by Supdami $\mathrm{W}$ et al., due to the high cost charged for the treatment. ${ }^{14}$

The General Ward was preferred by most patients when it came to Ward selection $(63.18 \%)$. In a similar study conducted by Supdami W et al., $71.86 \%$ of patients preferred the class-III ward. The findings demonstrate the patient's burden for the amount to be spent on treatment, which is also due to factors such as the cost charged for various types of wards. ${ }^{14}$ The estimated costs in this study are the direct costs of hospitalization and do not include costs such as travel, lodging, food, and so on. Only in $35.91 \%$ of cases were the treatment costs covered by the patient's or employer's insurance, with the remaining $64.09 \%$ cases borne by the patient's families. The findings were similar to the study conducted by Kumar $\mathrm{N}$ et al., in the rural district of Karnataka \& found that $77.6 \%$ of households used their savings to pay for dengue care, $18.4 \%$ borrowed money from friends and family to pay for hospital bills, and the remaining $3 \%$ used their health insurance as a financial tool. ${ }^{15}$

\section{Conclusion}

The study concludes that male patients were predominant than female patients. The majority of the patients were seen in 19-30 years. The mean length of hospital stay was 4.55 days. The median total direct medical costs was USD 119.89 . The laboratory charges incurred $34.02 \%$ of the total costs, consultation charges incurred $17.18 \%$ of the total costs, and medication charges incurred $14.72 \%$ of the total costs. Male 
Table 4

Categorization of cost based on age groups, gender, healthcare scheme and wards.

\begin{tabular}{|c|c|c|c|c|c|}
\hline \multicolumn{5}{|l|}{ Categorization of cost based on gender } & \multirow[t]{3}{*}{ p-value } \\
\hline \multicolumn{3}{|c|}{ Total Costs (USD) } & \multicolumn{2}{|c|}{ Total Costs (INR) } & \\
\hline & Median & IQR (Q3-Q1) & Median & IQR (Q3-Q1) & \\
\hline Gender & & & & & 0.951 \\
\hline Male & 102.83 & $142.93-68.97$ & 7495 & $10417.50-5026$ & \\
\hline Female & 99.16 & $134.73-75.77$ & 7227.50 & $9820-5522.50$ & \\
\hline \multicolumn{6}{|c|}{ Categorization of cost based on age groups } \\
\hline Age & & & & & 0.001 \\
\hline $19-30$ & 93.04 & $129.50-63.06$ & 6780 & $9437.50-4522.50$ & \\
\hline $31-45$ & 101.43 & $137.29-69.39$ & 7392 & $10005-5057$ & \\
\hline $46-60$ & 135.16 & $175.50-98.18$ & 9850 & $12790-7155$ & \\
\hline \multicolumn{6}{|l|}{ Categorization of cost based on wards } \\
\hline Wards & & & & & 0.001 \\
\hline General ward & 80.61 & $103.60-58.11$ & 5875 & $7550-4235$ & \\
\hline Semi special ward & 141.54 & $204.87-108.27$ & 10315 & $14930-7890$ & \\
\hline Room with TV & 152.38 & $196.57-117.94$ & 11105 & $14325-8595$ & \\
\hline Room with AC & 160.22 & $218.92-121.29$ & 11676 & $15955-8840$ & \\
\hline \multicolumn{6}{|c|}{ Categorization of cost based on healthcare scheme } \\
\hline Scheme & & & & & 0.001 \\
\hline No Scheme & 104.01 & $141.53-68.54$ & 7581 & $10315-4995$ & \\
\hline Employees' State Insurance (ESI) & 85.41 & $121.50-74.78$ & 6625 & $8855-5450$ & \\
\hline Sampoorn Suraksha Insurance (SMI) & 78.41 & $99.20-58.86$ & 5715 & $7230-4290$ & \\
\hline Kshema Health Card (KHC) & 110.93 & $134.78-89.03$ & 8085 & $9825-6490$ & \\
\hline Arogya Bhagya Yojane (ARB) & 123.56 & $135.12-123.53$ & 9005 & $9850-9005$ & \\
\hline Student Scheme & 146.06 & $196.51-73.83$ & 10645 & $14325-5382$ & \\
\hline Med Save Health Insurance (MED) & 207.91 & $234.80-159.65$ & 15152.5 & $17118.5-11640$ & \\
\hline Sevanjali Health Insurance (SVT) & 207.91 & $90.98-90.98$ & 15152.5 & $6633-6633$ & \\
\hline
\end{tabular}

patients had a higher total median direct cost USD 102.83 than females USD 99.16, and the age group 46-60 years had the highest total median direct costs USD 135.16 when compared to other age groups.

\section{Funding}

There was no specific grant for this research from any funding agencies.

\section{Declaration of competing interest}

The authors state that they have no conflicts of interest.

\section{Acknowledgement}

We would like to express our gratitude to the NGSM Institute of Pharmaceutical Sciences, Nitte (Deemed to be University), Mangaluru for providing the research facilities for this study.

\section{References}

1 Dengue and severe dengue-World Health Organization fact sheet. Available from: https://www.who.int/news-room/fact-sheets/detail/dengue-and-severe-dengue. [Last cited on 4 May, 2021].

2 Hariharan D, Das MK, Shepard DS, Arora NK. Economic burden of dengue illness in India from 2013 to 2016: a systemic analysis. Int J Infect Dis. 2019;84(S):S68-S73.

3 National Guidelines Dengue Case Management during Covid-19 Pandemic. National Vector Borne Disease Control Programme; 2020. Available from https://nvbdcp.gov.
in/Doc/National\%20Guideline\%20for\%20Dengue\%20case\%20management $\% 20$ during\%20COVID-19\%20pandemic.pdf [Last cited on 4 May, 2021].

4 Tiwari S, Shukla MK, Chand G, et al. Outbreak of dengue in Central India in 2016: clinical, laboratory and epidemiological study. Indian J Med Res. 2019;150(5): $492-497$.

5 Singh J, Dinkar A, Atam V, et al. Awareness and outcome of changing trends in clinical profile of dengue fever: a retrospective analysis of dengue epidemic from January to December 2014 at a tertiary care hospital. J Assoc Phys India. 2017;65(5); 42-46.

6 Mateti UV, Nagappa AN, Mateti KV. Pharmacoeconomics: the missing link in health care system. J Pharmaceut Res. 2011;10(1):10-13.

7 Constenla D, Armien B, Arredondo J, et al. Costing dengue fever cases and outbreaks: recommendations from a costing dengue working group in the Americas. Value Health Reg Issues. 2015:80-91.

8 Scaria S, Raju R, Joseph S, Mohan A, Nair AA. Pharmacoeconomics: principles, methods and Indian scenario. Int J Pharmaceut Sci Rev Res. 2015;34(1):37-46.

9 Jain D, Rajput R, Pathak V, Mittal A, Jain P. Changing trends in clinical presentation and biochemical spectrum of dengue fever: an observation of a tertiary care centre. Arch Clin Infect Dis. 2017;12(3), e6221.

10 Dinkar A, Singh J. Dengue infection in North India: an experience of a tertiary care center from 2012 to 2017. Tzu Chi Med J. 2020;32(1):36-40.

11 Kale AV, Haseeb M, Reddy CS, Khan S, Golwelkar A, et al. Clinical profile and outcome of dengue fever from a tertiary care center at Aurangabad Maharashtra, India: an observational study. IOSR J Dent Med Sci. 2014;13(9):14-19.

12 Vo NTT, Phan TND, Vo TQ. Direct medical costs of dengue fever in Vietnam: a retrospective study in a tertiary hospital. Malays J Med Sci. 2017;24(3):66-72.

13 Panmei K, Joseph AK, Rose W, et al. Direct cost of illness for dengue in hospitalized children and adults at a referral hospital in India. Int J Infect Dis. 2019;84(S): S64-S67.

14 Supdami W, Izzah QN, Suwantika AA, Perwitasari DA, Abdulah R. Cost of illness study of patients with dengue hemorrhagic fever at one of the private hospitals in Yogyakarta. J Pharm Bioall Sci. 2019;11(S):S587-S593.

15 Kumar N, Shyam AC, Vidya. Cost burden of dengue fever in a rural Ramanagara district of Karnataka state. J Med Sci. 2016;2(4):59-61. 